\title{
Construction and evaluation of physical fitness training system for high level Dragon Boat athletes
}

\author{
Hong-chun Jia ${ }^{1}$, Song-bo $\mathrm{He}^{1, *}$, Hao-peng Zhang ${ }^{2}$ \\ ${ }^{1}$ Beihua University ,Jilin, China \\ ${ }^{2}$ Beihua University, College of physical education, Longteng innovation and entrepreneurship education \\ base \\ *Corresponding author:Song-bo He
}

Keywords: Dragon Boat, Athletes, Physical fitness, Training, Evaluation, Construction.

\begin{abstract}
. at present, the main problems faced by dragon boat athletes are mainly lack of attention to physical training, lack of scientific and technical degree of physical training and lack of professional degree. According to the principle of testability, the principle of systematicness and the principle of independent objectivity, it is the basic principle to evaluate the physical training system of high level dragon boat athletes. By forming the basic principles of standardized physical training, the theoretical basis and practical application of scientific physical training is an effective way to build physical training system of dragon boat sports.

Dragon boat race is one of the traditional national sports events in China. It has a history of more than two thousand years ago. As the birthplace of dragon boat movement, China has gradually formed a modern competitive sports on the basis of carrying forward the traditional culture, and has been popularized all over the world. More and more people pay attention to the indomitable fighting spirit, perseverance and striving for the spirit of dragon boat race. With the continuous development of competitive sports, dragon boat racing has received attention and rapid development enough in parts of Asia and Europe and other countries, China's traditional dragon boat competition level faces important challenges. Therefore, according to the current situation of Dragon Boat Sports in China, it is an important task for the Dragon Boat Sports Innovation and development to draw up the special strength training system and evaluation criteria of the high level dragon boat athletes.
\end{abstract}

\section{Dragon Boat athlete physical training faces the main question}

(1) Insufficient attention is paid to physical training

Physical fitness training is an important basic content for Dragon Boat athletes in comprehensive training. However, in the current investigation and research process, the thought of "emphasizing technology and reducing physical fitness" still exists in the competitive training of dragon boat race. According to the survey, the Dragon Boat Sports Training in China focuses on the rowing technique, while the physical training research is relatively few. The systematic training for physical fitness is not enough in-depth. At the same time, dragon boat training has periodicity in training time and training intensity is limited to a certain extent, in the process of training including training plan, strength and other aspects have a relatively perfect system of training program, often resulting in special training effect is not prominent. In the competitive arena of dragon boat race, it is difficult to achieve the overall level leap because of lack of physical capacity.

(2) The degree of physical fitness training is not enough

With the rapid development of science and technology in China, the monitoring of athletes' physical fitness through modern means of science and technology is an important trend in the development of competitive sports. The research found that, in the training process of China's dragon boat through scientific means of monitoring the level of physical fitness and other sports is not enough developed slightly. Through the use of relevant physiological and biochemical 
instruments can timely and effectively monitor the level of athletes' performance, can provide important data support for athletes' physical training, so as to achieve good training effect. In addition, in the training process of daily training occurred often leads to excessive and blind training phenomenon, make full use of the advanced monitoring instrument for physical detection helps to find out the physical training mode for Dragon Boat athletes most effective, fast and helps to improve the athletes' personal fitness dragon boat.

(3) Lack of specialization

Although Dragon Boat Sports originated in China, the professional level of dragon boat movement is relatively slow. In China, dragon boat race is not a key project for the development of competitive sports. Therefore, it is weak in system guarantee and so on. Through the investigation, it is found that the Dragon Boat Sports members are more composed of dragon boat lovers, and their professional level is low. At the same time, the Dragon Boat Sport from the traditional national sport to the occupation sport relatively late, specialized for the Dragon Boat Sport Fitness is relatively imperfect, at present mostly refers to other sports for physical training, due to the different movement of the physical features and motion mechanism is also different, which seriously affected the effect of physical training of dragon boat athletes. Through professional training, it is an important problem for Dragon Boat Sports to make a relatively perfect physical training method for Dragon Boat sports.

\section{Design principles of physical fitness evaluation system for Dragon Boat Sports}

(1) Principle of testability

Testability is one of the most important principles in the design of evaluation system. Testability refers to the basic indicators that can directly or indirectly measure the physical fitness in Dragon Boat Sports. It is easy to give relevant evaluation opinions through the measurement of evaluators. The selection process evaluation index in considering testability at the same time, to meet the evaluation index system can comprehensively reflect the purpose of the evaluation, it can be observed at the same time also should be taken into consideration as far as possible to choose the best, quantitative indicators are simplified. In addition, when the evaluation index can be quantitative or qualitative judgment, priority should be given to quantitative judgment, and the reliability of the evaluation index can be guaranteed on the basis of meeting the testability principle of the data.

(2)Systematic principle

The system refers to the principle of the whole process of dragon boat athletes in special training to dragon boat sport life ended, according to the inherent law of human physical development, formulate the corresponding evaluation index system, the completion of the Dragon Boat athletes to monitor and improve. In the development of physical evaluation system of process to focus and follow the principle of comprehensive, set fitness evaluation index in different training stages, the Dragon Boat athletes in various stages of physical training to give effective guidance in the process to be focused, reducing unnecessary work, seize the key index of each stage are according to the monitoring and regulation of the focus on the overall development, to improve the whole.

(3) The principle of independent objectivity

The principle of independent objectivity is an important principle for the design of physical fitness evaluation system of dragon boat sports. In the process of setting up the evaluation index of physical fitness, the principle of pursuing knowledge is scientific, and the index selection is made. In the process of formulating, there exists the problem of relative overlap of indexes. Therefore, in the process of formulating index monitoring, according to the actual situation, the independent indicators should be formulated, and the indexes should not be overlapped as much as possible. Each index has independent evaluation criteria and contents. At the same time, the evaluation index should follow the principle of objectivity, grasp the evaluation index system in the composition and connotation of a more correct direction, can timely feedback objective essence. The objective, comprehensive and extensive selection requirements should be achieved in the process of formulating evaluation system objectives. 


\section{Construction of physical training for Dragon Boat Sports}

Dragon Boat sports fitness training is an important guarantee for the best condition of athletes. Therefore, the formation of scientific Dragon Boat physical training is the key content of dragon boat training. The physical fitness content of dragon boat training mainly includes: sports function, sports quality and body shape quality. The training objects of motor function mainly include the regulation and training of skeletal muscle system, energy metabolism system and nerve conduction system. Sports quality mainly includes special strength quality, special speed quality, special endurance quality, special flexibility quality and special sensitive quality and other different angles of training.

(1) To form the basic principles of standardized physical training

In the daily Dragon Boat physical training process, the main forms of training are divided into endurance and flexibility training, speed training, strength training, sensitivity training and core strength training. In the training process, the standardized scientific training method is an important guarantee to improve the technical level effectively. By means of scientific training rules, it is the main way of dragon boat training practice to use modern technical means. To develop and adjust the training plan according to the actual situation of dragon boat, reasonable training, analysis and judgment through the training effect of training data index of the dragon boat, which effectively improve the effect of physical training, physical fitness training to achieve the best results of.

(2)The theoretical basis and practical application of scientific physical fitness training

Physical training is the power supply by the human body energy substance, from the theoretical analysis of biological energy supply system can effectively improve the level of the effect of physical training, through energy analysis theory can enhance the level of physical training effects of the establishment of scientific. At the same time, the dynamic mechanism is an important theoretical basis for physical training. In the training process, the Dragon Boat athletes can carry out the strength training according to the mechanical principle of human movement, so that the training effect is improved significantly. In the training process, a fast moving ability training, improve the ability of rapid completion of the body movement, on the other hand, the sustained and rapid movement ability training, ability training to achieve sustained and rapid completion of the anaerobic respiration conditions. At the same time, rapid response ability training, Dragon Boat athletes can use physical training to strengthen such sports reaction ability.

\section{Acknowledgements}

This paper is a research project of Beihua University students' innovation project, horizontal topi c: Dragon Boat land training equipment (NO:201602014).

\section{References}

[1] Lin Yaohui. Dragon boat training skills and strength training [J]. adolescent sports, 2017, (05): 50-51.

[2] He Fei, Wang Xiaogang. Research on physical training methods and means of high level dragon boat racing athletes [J]. Hubei sports science and technology, 2017,36 (02): 142-144.

[3] Li Li. Screening and classification of special strength indicators for elite college Dragon Boat athletes [D]. Northeast Normal University, 2016.

[4] Zhang Liangzhi, Li Li, Wang Wei. Significance, deficiency and trend of special strength research on College Dragon Boat athletes [J]. new curriculum (middle), 2015, (01): 221.

[5] Zhang Peng. Special features and winning rules of dragon boat racing [J]. contemporary sports science and technology, 2011,1 (01): 1-2.

[6]Shen Jiaqi. Review of the research status of Competitive Dragon Boat Sports Technology and physical fitness theory in China [J]. Journal of Tongren University, 2010,12 (03): 58-59+122. 